\title{
A Rare Anomalous Artery Originating From Axillary Artery
}

\author{
T. Sobha Devi ${ }^{1}$, P.Hari Krishna ${ }^{2}$, J. Sumalatha ${ }^{3}$, \\ ${ }^{I}$ Assistant professor, Department of Anatomy, S.V.Medical college, Tirupathi, India \\ ${ }^{2}$ Assistant professor, Department of E.N.T, S.V.Medical college, Tirupathi, india \\ ${ }^{3}$ Postgraduate Department of Anatomy, S.V.Medical college, Tirupathi, India
}

\begin{abstract}
During routine dissections in the Dept. Of anatomy, Sri Venkateswara Medical college, Tirupathi, A.P. we encountered a rare anomaly in an adult male cadaver on the right upper limb. The third part of the axillary artery unilaterally divides into two major arterial stems, named according to their localization as deep brachial artery and superficial brachial artery (brachial artery proper).Deep brachial artery passes at first in between the two roots of median nerve, and later deep to lateral root of median nerve. The deep brachial artery gives off the posterior circumflex humeral artery, anterior circumflex humeral artery, subscapular artery, and profunda brachii artery and lower down in the arm, it terminates by dividing into superior ulnar collateral artery and inferior ulnar collateral artery. This case is a anamoly of the axillary artery that has been rarely documented in the literature(0.12-3.2\%). Accurate knowledge of the normal and variant arterial anatomy of the axillary artery is important for surgeons and specialists using radiodiagnostic techniques. The improved knowledge would allow more accurate diagnostic interpretations and surgical treatment.
\end{abstract}

Key words: Axillary artery, superficial brachial artery, deep brachial artery.

\section{Introduction}

The third part of the axillary artery unilaterally divides into two major arterial stems, named according to their localization as deep brachial artery and superficial brachial artery (brachial artery proper). This case is a anamoly of the axillary artery that has been rarely documented in the literature(0.12-3.2\%). Accurate knowledge of the normal and variant arterial anatomy of the axillary artery is important for surgeons and specialists using radiodiagnostic techniques. The improved knowledge would allow more accurate diagnostic interpretations and surgical treatment.

\section{Material And Methods}

During routine dissections in the Dept. Of anatomy, Sri Venkateswara Medical college, Tirupathi, A.P in between the years 2006-2012, we encountered a rare anomaly in an embalmed adult male cadaver on the right upper limb.Axillary artery traced in the axilla of both limbs, Superficial brachial artery and deep brachial artery traced until the cubital fossa, Arteries are painted, photographs taken and labelled.

\section{Observations}

The third part of the axillary artery unilaterally divides into two major arterial stems, named according to their localization as deep brachial artery and superficial brachial artery (brachial artery proper).Deep brachial artery passes at first in between the two roots of median nerve, and later deep to lateral root of median nerve. The deep brachial artery gives off the posterior circumflex humeral artery, anterior circumflex humeral artery, subscapular artery, and profunda brachii artery and lower down in the arm, it terminates by dividing into superior ulnar collateral artery and inferior ulnar collateral artery. The superficial brachial artery runs its normal course in the arm, gave muscular branches in the arm, divided into radial and ulnar arteries in the cubital fossa. Fig 1, 2, 3 showed observations. Posterior circumflex humeral artery left the axilla along with axillary nerve through quadrangular space. Profunda brachii artery entered the back of arm with radial nerve. Superior ulnar collateral artery and inferior ulnar collateral artery participated in the formation of anastamosis around the elbow infront and behind the medial epicondyle. Superficial brachial artery atfirst lie medial to median nerve later in the middle of arm it crossed lateral to it.Left sided axillary artery was normal in its course relations and branching pattern. 


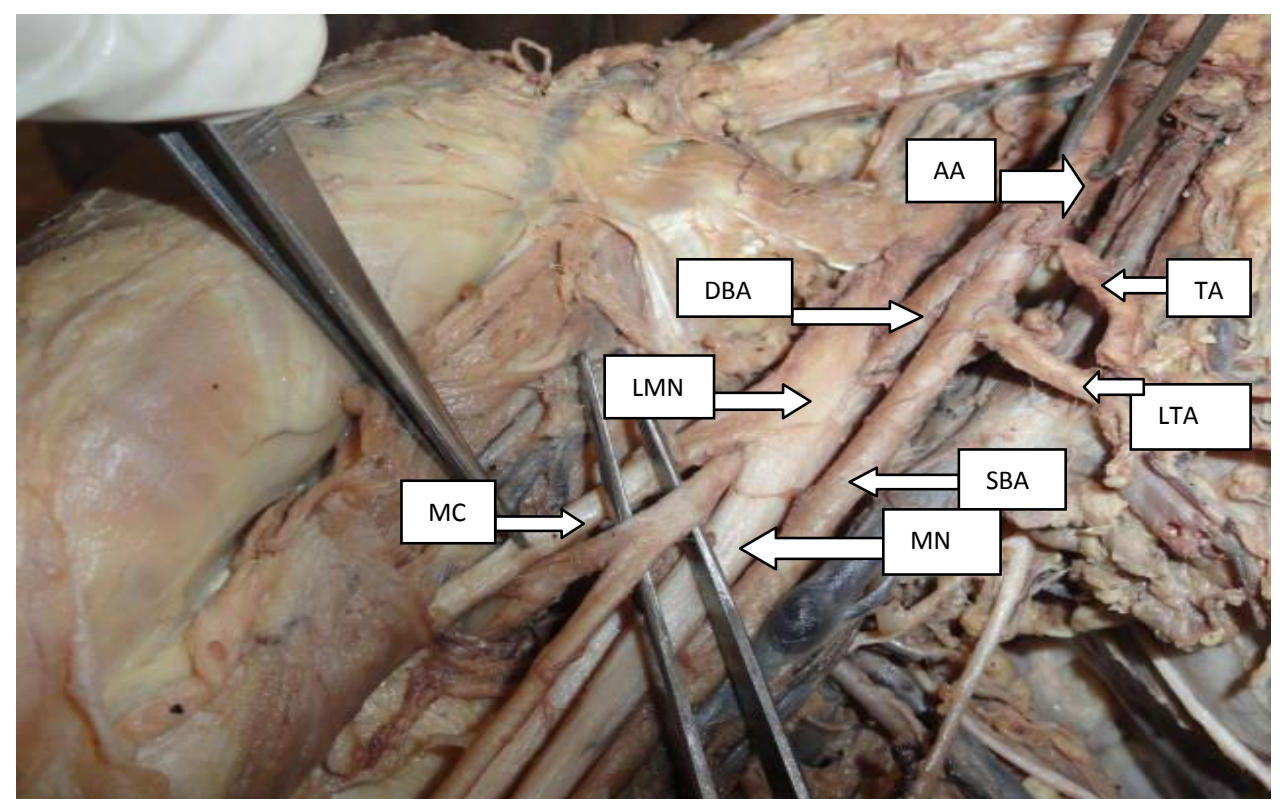

FIGURE.1 Before painting the artery.Probed artery is DBA passing deep to the lateral root of MN

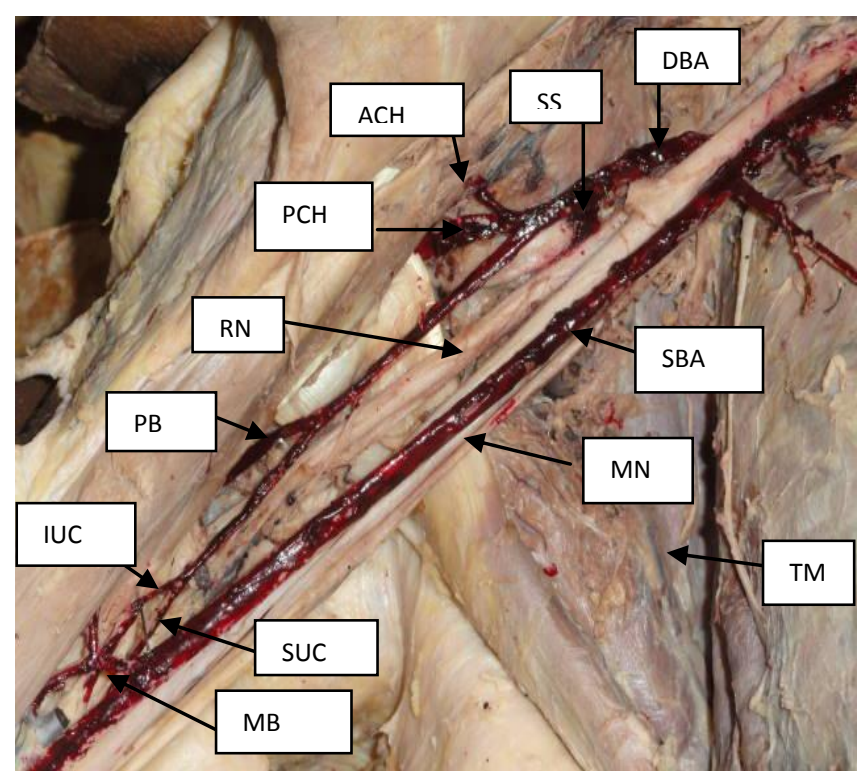

FIGURE. 2 Shows branches of SBA \& DBA

representation

TA: Thoraco acromian artery

DBA: Deep brachial artery

MCN: Musculocutaneous nerve

LT: Lateral thoracic artery

SBA: Superficial brachial artery

SS: Subscapular artery

PCH: Posterior circumflex humeral PB: Profunda brachii artery

SUC: Superior ulnar collateral artery MB :Muscular branches

$\mathrm{RN}$ : Radial nerve

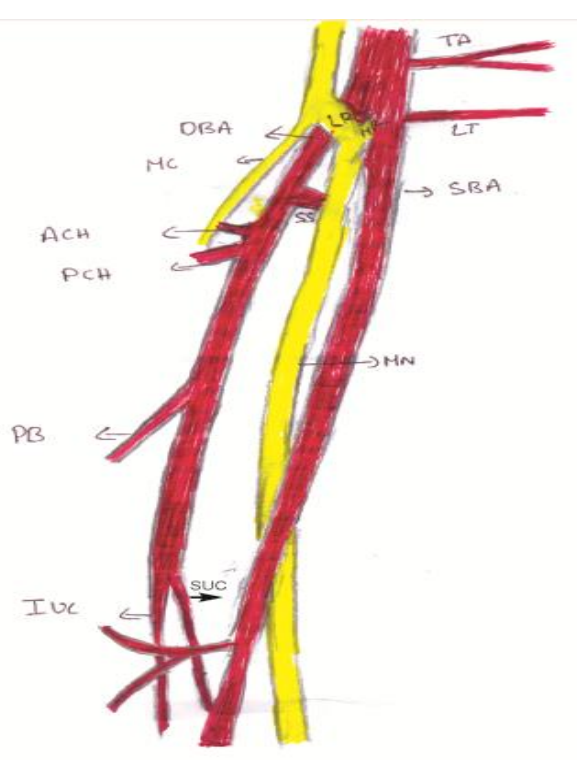

FIGURE. 3 Shows schematic

LMN: Lateral root of median nerve

MN: Median nerve

ACH: Anterior circumflex humeral artery IUC: Inferior ulnar collateral artery

TM: Teres major muscle

\section{Discussion}

According to Cavdar S, et al (ref 1) The third part of the axillary artery unilaterally divides into two major arterial stems, named according to their localization as deep brachial artery and superficial brachial artery (brachial artery). The deep brachial artery gives off the posterior circumflex humeral artery, anterior circumflex humeral artery, subscapular artery, and profunda brachii artery. It continues its course in the arm lateral to the median nerve and terminates by giving a minute twig to the radial artery. The superficial brachial artery is larger in caliber than the deep brachial artery and gives no branches in the arm region. In the cubital fossa it gives the ulnar and the radial arteries.In the present variation the deep brachial artery has given in addition to the above, Superior ulnar collateral artery, Inferior ulnar collateral artery. Superficial brachial artery has given muscular branches in the arm.According to Nakatani T, et al (ref 3) axillary artery passed inferior and dorsal to the medial 
cord.The superficial brachial artery descended ventral to the median nerve and divided into the radial and ulnar arteries in the cubital fossa.Present anomaly showed the abnormal artery passing deep to the two roots of median nerve and the Superficial brachial artery passed ventral to the 2 roots of median nerve. The Present variation is very rare and incidence is around $0.12-3.2 \%$ in the available literature. Axillary artery is the remnant of proximal part of axis artery of upper limb bud. Axis artery of upper limb developes from lateral branch of seventh inter segmental artery (ref.6). Origin of anomalis in the branching pattern of the upper limb arteries was attributed to defex in the embryonic development of upper limb arteries. Arrest at any stage of development showing regression, retension or reappearance may produce various arterial variations and course of limb arteries (ref.7). The normal and abnormal anatomy of the axillary region is having practical importance for the vascular radiologist and surgeon and it should be known for accurate diagnostic interpretation. The anatomical knowledge of the axillary region is of crucial importance for neurosurgeons and specialists using the radiodiagnostic techniques, particularly in cases involving traumatic injuries.Axillary artery variations are taken into consideration in procedures such as treating Axillary artery aneurysms, Radical mastoidectomy, axillary artery thrombosis, Brachial plexus block, Axillary artery catheterisation, Surgical intervention in shoulder dislocation and fracture of upper end of humerus.

\section{References}

[1]. Cavdar S, Zeybek A, Bayramiçli M Rare variation of the axillary artery. Clin Anat. 2000;13(1):66-8. PMID: 10617889.

[2]. Kachlik D, Konarik M, Baca V. Vascular patterns of upper limb: an anatomical study with accent on superficial brachial artery. PMID: 21342134.

[3]. Nakatani T, Mizukami S, Tanaka S A rare case: the medical cord of the brachial plexus sandwiched between the axillary and superficial brachial arteries. Kaibogaku Zasshi. 1997 Apr;72(2):151-4. PMID:9153967.

[4]. VijayaBhaskar P, Ritesh R, Shankar PR. Anomalous branching of the axillary artery: a case reportKathmandu Univ Med J (KUMJ). 2006 Oct-Dec;4(4):517-9. PMID: 18603967.

[5]. Yang HJ, Gil YC, Jung WS, Lee HY. Variations of the superficial brachial artery in Korean cadavers. J Korean Med Sci. 2008 Oct;23(5):884-7. PMID: 18955798

[6]. Asim kumar data. The circulatory system, in: Human Embryology, $5^{\text {th }}$ edition. Current books international may 2005;195-197.

[7]. Hamilton WJ, Mossman HW. Cardiovascular system, in: Human Embryology, $4^{\text {th }}$ edition. Baltimores, Williams and Wilkins, $1972 ; 271-290$. 